\title{
Apports des traçages artificiel et naturel à la connaissance des circulations et des zones de recharge en région semi-aride: cas de l'Atlas de Beni Mellal (Maroc) \\ Contribution of artificial and natural tracers to the knowledge of flow and recharge in a semi-arid region: the case of Beni Mellal Atlas (Morocco)
}

\author{
L. Bouchaou, P. Chauve, J. Mania, J. Mudry et J. L. Michelot
}

Volume 8, numéro 1, 1995

URI : https://id.erudit.org/iderudit/705212ar

DOI : https://doi.org/10.7202/705212ar

Aller au sommaire du numéro

\section{Éditeur(s)}

Université du Québec - INRS-Eau, Terre et Environnement (INRS-ETE)

ISSN

0992-7158 (imprimé)

1718-8598 (numérique)

Découvrir la revue

Citer cet article

Bouchaou, L., Chauve, P., Mania, J., Mudry, J. \& Michelot, J. L. (1995). Apports des traçages artificiel et naturel à la connaissance des circulations et des zones de recharge en région semi-aride: cas de l'Atlas de Beni Mellal (Maroc). Revue des sciences de l'eau / Journal of Water Science, 8(1), 43-55.

https://doi.org/10.7202/705212ar

\section{Résumé de l'article}

Pour mieux comprendre les modalités d'alimentation des sources de l'Atlas, des campagnes de coloration et d'analyses isotopiques ont été effectuées sur l'ensemble des sources. La disparité des circulations, la variabilité des vitesses et des taux de restitution, révèlent un aquifère complexe ou à plusieurs entrées. La majorité des écoulements convergent vers la source de Ain Asserdoune qui constitue une zone d'abaissement d'axe de plis. L'étude isotopique montre la variation des teneurs en 180 avec l'altitude des zones de recharge des différents exutoires et avec la saison. Les gradients définis autorisent la détermination de l'altitude moyenne des impluviums des systèmes jusqu'alors mal connus, en particulier celui de l'exutoire principal du massif, Ain Asserdoune. 


\title{
Apports des traçages artificiel et naturel à la connaissance des circulations et des zones de recharge en région semi-aride : cas de I'Atlas de Beni Mellal (Maroc)
}

\author{
Contribution of artificial and natural tracers to the knowledge \\ of flow and recharge in a semi-arid region: \\ the case of Beni Mella「 Atlas (Morocco)
}

L. BOUCHAOU1', P. CHAUVE2 2 , J. MANIA2, J. MUDRY2 et J.L. MICHELOT3

Reçu le 22 mars 1994, accepté le 3 novembre 1994*.

SUMMARY

The chalky massif of Beni-Mellal, which supplies many springs, spreads over a $365 \mathrm{~km}^{2}$ area of 500 to 2,400 meters elevation (average altitude, $1,200 \mathrm{~m}$ ). Rainfall in this sector varies between $\mathbf{4 0 0} \mathbf{~ m m}$ and $700 \mathrm{~mm}$ /year. All springs emerge along the major coastal fautt giving the Liassic massif overthrushing the Cenozoic lands of piemont. The most important is Ain Asserdoune spring with an average discharge of about $1.1 \mathrm{~m} 3 / \mathrm{s}$. The mainly chalky and dolomitic formations are very fractured according to preferential directions (N150-180 and N50-70) and show some karstification features at the surface. Some tracing experiments with fluorescein allowed the delimitation of the boundaries of the intake area of Ain Asserdoune Basin and the determination of the groundwater flow velocity. Two types of flow were identified with fast (100 to $231 \mathrm{~m} / \mathrm{h}$ ) and slow circulation $(<100 \mathrm{~m} / \mathrm{h}$ ). Most of circulations converge towards the spring of Ain Asserdoune (fig. I) with an important intake area. This convergence results from an axis lowering. The variability of the circulation axes, the velocity and the rate of restitution suggest either a complex aquifer or an aquifer with several inputs.

Two water samplings (the first for the Beni Mellal Atlas) were carried out in March and in September 1993 on the several springs, in order to study the variations of the oxygen-18 isotope. The oxygen-18 contents range between $-5.5 \%$ and $-7.7 \%$ (table 2 ). The most depleted waters directly exit from the Liassic aquifer or emerge at high altitudes; those which are richer in oxygen-18 emerge at the level of foothills, either from the Tertiary or from Liassic scales. The relationship between the oxygen-18 and the $\mathrm{H}-2$ content of some samples indicates that the points are aligned along the world meteoric line (Craig, 1961). Even if the waters of Dir (piemont) springs are slightly evaporated because they partly originate in seeping Liassic waters, oxygen-18 content is probably mainly

1. Labo. Géol. Appl. Faculté des Sciences, BP S/28, 80000 Agadir, Maroc.

2. Labo. Géol. Struct et Appl. 1 place Leclerc, 25000 Besançon, France.

3. Labo. d'Hydrologie et de Géochimie isotopique, Bat. 504, 91504 Orsay Cedex, France.

* Les commentaires seront reçus jusqu'au 15 novembre 1995. 
controlled by an altitude effect. Oxygen-18 data for local precipitations are not available; however, a local relationship between oxygen-18 content and mean recharge altitude was etablished for eleven (11) springs with small recharge areas whose mean altitude may be estimated from topography and geology. Oxygeneighteen ratio is very well correlated to recharge average elevation for spring samples $(\delta \% \circ(O-18)=-3-0,0026 \times$ altitude, $r=0,96 ; f i g .3)$, when it is not for autumn samples. This gradient is very close to the regional relationship proposed by Marce (1975) and Kabbaj et al. (1978) ( slope : 3\%o in oxygen-18 per $1,000 \mathrm{~m}$ ). This difference is attributed to the seasonal variations in the origin of water that feeds the springs. In the high water season, the whole basin area contributes to the recharge. In contrast, during low water period, water mainly originates from altitudes higher than the mean altitude of the basin, probably with a longer transit time. The seasonal variations are particularly marked for the Dir (piemont) springs (with a lower flux and intake area range of 800 to 1,200 m). They are weaker for the Lias springs and even non-existent for the main spring of the massif, Ain Asserdoune. In this latter case, the smoothing $(-7.6 \% 0$ and $-7.5 \%$ ) of seasonal variation ( which is also observed for temperature : 15.5 to $16^{\circ} \mathrm{C}$ ) gives evidence of important water reserves. A mean recharge altitude higher than 1,700 m may be proposed for Ain Asseroune.

Based on this analysis, some emergences which in the part were assigned to the same hydrologic system can now be discriminated. On the other hand, with a similar value of oxygen-18 ratio, rather distant springs can be considered as belonging to the same hydrogeological Atlasic unit. So the relationship which was quantified ( slope : $2.6 \%$ in oxygen-18 per $1,000 \mathrm{~m}$ ) is usable for the whole Atlasic range on account of regionaly similar weather. The methodology selected in this work corroborates the results obtained by hydrometric analysis concerning the very important groundwater reserves in the Beni Mellal aquifer.

Key-words : tracer, spring, velocity, aquifer, environmental isotope, intake area, gradient, recharge atitude, reserves.

RÉSUMÉ

Pour mieux comprendre les modalités d'alimentation des sources de l'Atlas, des campagnes de coloration et d'analyses isotopiques ont été effectuées sur l'ensemble des sources. La disparité des circulations, la variabilité des vitesses et des taux de restitution, révèlent un aquifêre complexe ou à plusieurs entrées. La majorité des écoulements convergent vers la source de Ain Asserdoune qui constitue une zone d'abaissement d'axe de plis. L'étude isotopique montre la variation des teneurs en 180 avec l'altitude des zones de recharge des différents exutoires et avec la saison. Les gradients définis autorisent la détermination de l'altitude moyenne des impluviums des systèmes jusqu'alors mal connus, en particulier celui de l'exutoire principal du massif, Ain Asserdoune.

Mots clés : traçage, source, vitesse, aquifere, isotope du milieu, altitude, recharge. bassin d'alimentation, gradient, réserves.

\section{1 - CARACTÉRISTIQUes de la RÉGION ÉtUdiée}


présente une superficie de $365 \mathrm{~km}^{2}$. La morphologie montre un étagement des altitudes entre $500 \mathrm{~m}$ (piémont appelé Dir) et $2400 \mathrm{~m}$ (montagne), avec une altitude moyenne de $1200 \mathrm{~m}$. Du point de vue climatique, la région reçoit une pluviométrie moyenne allant de $400 \mathrm{~mm}$ (plaine-piémont) à $700 \mathrm{~mm}$ (en montagne). Les chutes de neige varient d'une année à l'autre, mais en général elles accompagnent les pluies d'hiver et peuvent persister jusqu'à fin mars au delà de $1500 \mathrm{~m}$ d'altitude. La température moyenne est de l'ordre de $19^{\circ} \mathrm{C}$, les variations saisonnières sont importantes et l'amplitude journalière peut atteindre $20^{\circ} \mathrm{C}$. Les pluies efficaces sont de l'ordre de $100 \mathrm{~mm}$ sur la plaine et le Dir et de $300 \mathrm{~mm}$ en montagne. L'aquifère contenu dans ce massif donne naissance à de nombreuses sources (fig. 1 et tabl. 2) dont la plus importante est celle de Ain Asserdoune (Qmoy. $=1,1 \mathrm{~m}^{3} / \mathrm{s}$ ) qui constitue la ressource principale en eaux potable et agricole de la région. La structure montre un anticlinal ouvert qui chevauche vers le nord-ouest. Les différentes sources émergent en général au contact du chevauchement nord atlasique (fig. 1). Le système a fait l'objet d'études géologiques (MONBARON, 1982, 1985) et hydrogéologiques (BOUCHAOU, 1988). Les formations du Lias inférieur et moyen sont à dominante calcaire et dolomitique. L'étude de la fracturation a montré deux fourchettes directionnelles ( $N$ 150-180 et $N$ 50-70) qui peuvent guider probablement l'écoulement souterrain. Le faciès chimique des eaux est bicarbonaté calcique.

\section{2 - MÉTHOdES UTILISÉES}

Dans le cadre de ce travail, nous avons fait appel aux méthodes des traçages artificiel et naturel :

Des traçages à la fluorescéine ont été réalisés dans le massif afin de déterminer, le mode de circulation de l'aquifère (écoulement rapide ou retardé), le bassin d'alimentation de la source Ain Asserdoune et les relations avec les autres sources. En l'absence de perte localisée, cinq points d'injection répartis dans le massif ont été choisis (trous absorbants ou lits de ruisseaux). La surveillance des différentes sources et l'analyse des échantillons et des fluocapteurs révèlent la relation entre les points d'injection et les exutoires. Les taux et la forme des courbes de restitution renseignent sur les formes de décroissance plus ou moins rapide résultant de divers facteurs (dispersion hydraulique liée aux pertes de charge, diffusion, adsorption-désorption).

Une étude des teneurs en isotopes stables de l'eau, a été effectuée dans le but d'acquérir une meilleure connaissance des circulations et des zones de recharge.

Les analyses isotopiques ont été effectuées au laboratoire d'hydrologie et de géochimie isotopique d'Orsay (Université Paris sud) dans le cadre d'un stage financé par l'Agence Internationale de l'Energie Atomique (IAEA). Parmi les méthodes de l'hydrologie isotopique, celles fondées sur l'étude des 
Figure 1 Carte hydrogéologique de l'Atlas de Beni Mellal (points échantillonnés avec numéro).

Hydrogeological map of the Beni Mellal Atlas. (sampling point with number).

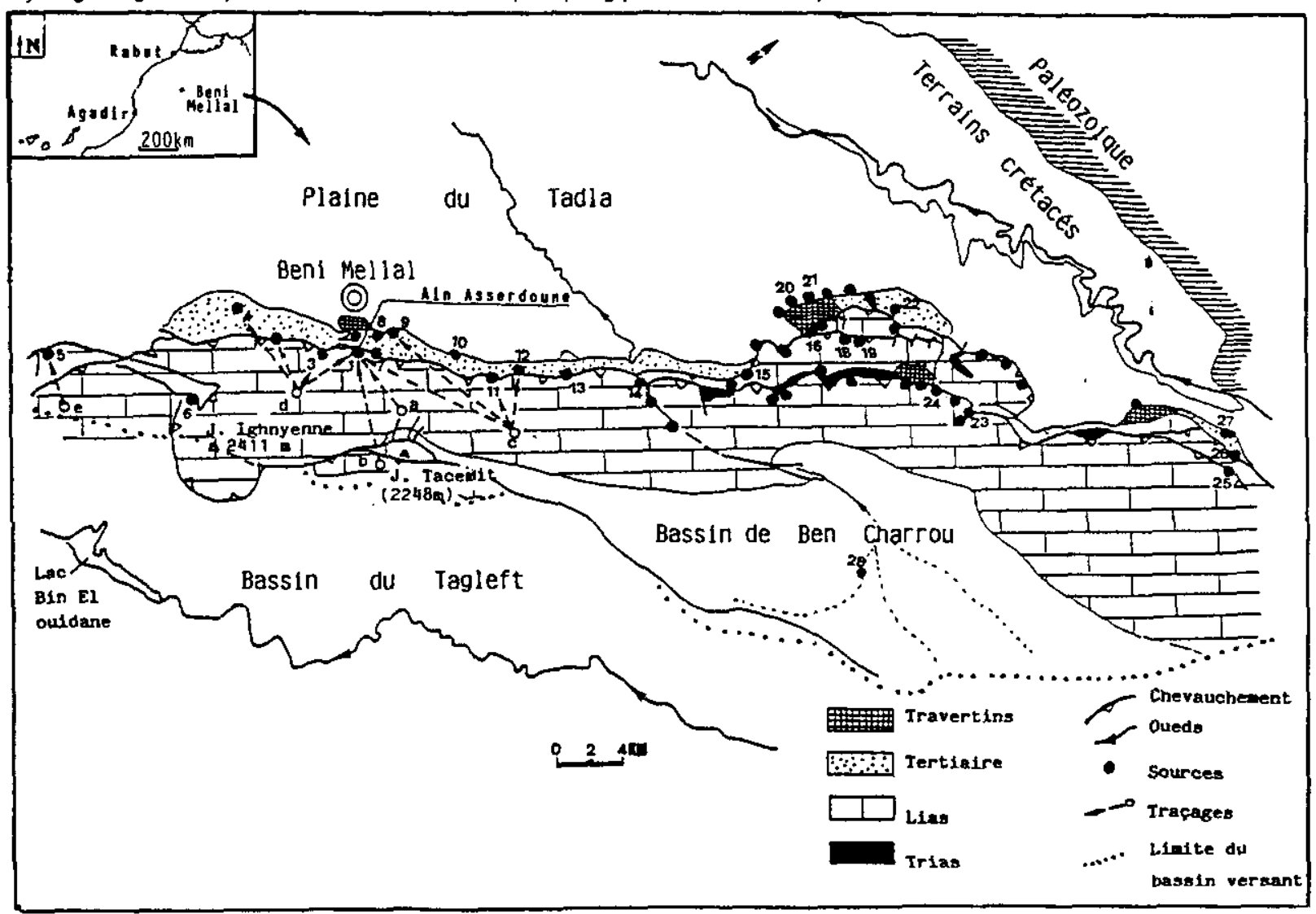


variations naturelles des teneurs en isotopes constitutifs de la molécule d'eau sont devenues d'un usage courant (FONTES, 1976 ; MOLINARI,1977 ; IAEA, 1981). Elles sont particulièrement adaptées aux régions caractérisées par de rapides et importantes variations d'altitude et/ou soumises à des conditions climatiques très marquées (ARANYOSSY, 1989), comme l'Atlas de Beni Mellal qui présente près de $2000 \mathrm{~m}$ de dénivelée. L'estimation des altitudes de recharge des aquifères est l'une des principales applications de ces méthodes (voir par exemple, pour les milieux karstiques, BAKALOWICZ et al., 1974 ; BLAVOUX et al., 1979 et 1990), qui revêt un intérêt particulier dans le cas de l'Atlas où les aires d'alimentatjon des sources sont mal connues. La variabilité saisonnière et spatiale aura un effet important dans l'utilisation des teneurs isotopiques pour la détermination des aires de recharge.

\section{RÉSULTATS ET INTERPRÉTATIONS}

\subsection{Traçage artificiel}

Les campagnes de coloration réalisées pour la première fois dans l'Atlas de Beni Melfal (BOUCHAOU et al., 1988) avaient pour but principal la délimitation du bassin d'alimentation de la source d'Ain Asserdoune. Sur la figure 1, on constate que la majorité des circulations convergent vers la source principale. Ce phénomène s'explique par le fait que la source se situe dans une inflexion du chevauchement nord atlasique (forme de gouttière) et sur un substratum triasique imperméable; ce qui justifie l'importance des débits. Ce mode de circulation lié à un abaissement d'axe a été déjà mis en évidence dans le Jura français (CHAUVE et al., 1986). La coloration (b) réalisée au delà de la limite topographique (fig. 1) indique un bassin hydrogéologique plus vaste que le bassin topographique.

Le tableau 1 résume les résultats des colorations effectuées. L'analyse de ces résultats montre deux types de circulations:

- des circulations rapides (100 à $231 \mathrm{~m} / \mathrm{h}$ ) qui s'expliquent par une mise en charge à l'occasion d'apports importants au système pendant les périodes de hautes eaux. Le débit de la source a relativement augmenté ( $900 \mathrm{l} / \mathrm{s}$ à $1000 \mathrm{l} / \mathrm{s}$ ). Ce transfert rapide et le taux de restitution élevé caractérisent des circulations dans un système de chenaux (MANGIN, 1975). La faible dilution implique l'absence de réservoirs le long du trajet: le passage du colorant peut être localisé à la surface de la zone noyée. Ceci s'explique par une circulation à travers un karst superficiel bien développé et une dénivellation importante $(600 \mathrm{~m})$;

- des circulations lentes avec des vitesses de circulations inférieures à $100 \mathrm{~m} / \mathrm{h}$ montrent que le colorant emprunte un réseau karstique mal développé en raison de l'absence d'un cheminement direct vers l'exutoire. Ce phénomène observé généralement en période d'étiage s'expliquait par le caractère peu transmissif de la zone noyée soit par un transit dans des systèmes annexes constituant les réserves du système. Durant les colorations réalisées en étiage, le débit est resté constant. 
Tableau 1 Résultats des traçages artificiels effectués dans le massif de Beni Mellal. Table 1 Results of artificial tracer in Beni Mellal massif.

\begin{tabular}{|c|c|c|c|c|c|c|c|c|}
\hline \multirow{2}{*}{ Coloration } & \multirow{2}{*}{$\begin{array}{l}\text { Coordonnées } \\
\text { du point } \\
\text { d'injection }\end{array}$} & \multirow{2}{*}{$\begin{array}{c}\text { Quantité } \\
\text { iinjectée } \\
\text { en (kg) }\end{array}$} & \multirow{2}{*}{$\begin{array}{l}\text { Points de } \\
\text { sorties }\end{array}$} & \multicolumn{3}{|c|}{$\begin{array}{c}\text { Vitesse des } \\
\text { circulations en } \mathrm{m} / \mathrm{h}\end{array}$} & \multirow{2}{*}{$\begin{array}{l}\text { Taux de } \\
\text { restitution }\end{array}$} & \multirow{2}{*}{ Observations } \\
\hline & & & & rapide & modale & lente & & \\
\hline a & $\begin{array}{l}X=418.000 \\
Y=191.000 \\
Z=1450\end{array}$ & 2,8 & $\begin{array}{l}\text { 1. Asserdoune } \\
\text { 2. Tamegnounte }\end{array}$ & $\begin{array}{r}231 \\
98\end{array}$ & $\begin{array}{c}125 \\
-\end{array}$ & $\begin{array}{l}22 \\
44\end{array}$ & $75 \%$ & $\begin{array}{l}\text { Injection dans un } \\
\text { aven avec un débit } \\
\text { de } 1 \mathrm{~V} / \mathrm{s}\end{array}$ \\
\hline b & $\begin{array}{l}X=425.000 \\
Y=186.000 \\
Z=1200\end{array}$ & 4,3 & 1. Asserdoune & 164 & 115 & 80 & $13 \%$ & $\begin{array}{l}\text { Injection dans le lit } \\
\text { d'un ruisseau } \\
(2 \mathrm{l} / \mathrm{s})\end{array}$ \\
\hline c & $\begin{array}{l}X=425.000 \\
Y=195.000 \\
Z=1250\end{array}$ & 2 & $\begin{array}{l}\text { 1. Asserdoune } \\
\text { 11. F. El ancer } \\
\text { 9. Ksabi }\end{array}$ & $\begin{array}{l}167 \\
125 \\
173\end{array}$ & - & - & - & $\begin{array}{l}\text { Injection au fond } \\
\text { d'une dioline dans } \\
\text { un volume d'eau de } \\
400 \text { । }\end{array}$ \\
\hline$d$ & $\begin{array}{l}X=410.000 \\
Y=190.500 \\
Z=1200\end{array}$ & 2,3 & $\begin{array}{l}\text { 1. Asserdoune } \\
\text { 3. Ourbiaâ } \\
\text { 4. Foum Oudi }\end{array}$ & $\begin{array}{l}229 \\
114 \\
188\end{array}$ & - & - & $17 \%$ & $\begin{array}{l}\text { Coloration suivie } \\
\text { d'une pluie cans un } \\
\text { trou absomant }\end{array}$ \\
\hline e & $\begin{array}{l}X=404.000 \\
Y=177.000 \\
Z=1100\end{array}$ & 0,3 & 5. Timoulilt & 50 & - & 10 & - & $\begin{array}{l}\text { Injection dans une } \\
\text { cartière }\end{array}$ \\
\hline
\end{tabular}

La dispersion des directions de drainage concorde avec les directions de fractures dominantes (N50-70 et N150-180). La forme des courbes de restitution montre généralement quelques pics en périodes de crue, alors qu'en étiage les courbes sont très étalées. Le temps de restitution du colorant dans les eaux de la source principale varie entre 6 et 11 jours. Toutefois, les variations des vitesses et des taux de restitution suggèrent un système à fonctionnement complexe et à perméabilité hétérogène.

La sortie du colorant au niveau d'autres sources indique aussi un écoulement dispersif et mal organisé, ce dont témoignent les nombreux exutoires sortant du système.

\subsection{Traçage naturel (Teneurs en Oxygène-18)}

Dans le but d'avoir plus de précision sur les bassins d'alimentation des différentes sources et de vérifier les résultats du traçage artificiel, deux campagnes de prélèvements pour analyses isotopiques ont été réalisées, l'une au mois de mars 1993 (hautes eaux) et l'autre au mois de septembre 1993 (basses eaux). L'échantillonnage de mars est effectué après un événe ment pluvieux, alors que celui de septembre est réalisé après une longue période sèche.

Sur l'ensemble des eaux analysées, les teneurs en oxygène-18 varient entre $-5,1 \%$ et $-7,7 \%$ vs SMOW (tabl. 2$)$. Les eaux les plus pauvres sortent directement de l'aquifère liasique ou émergent à de hautes altitudes, les plus riches sourdent en général au niveau du piémont, soit du Tertiaire soit des 
écailles liasiques (fig. 4). Les eaux des sources du Dir peuvent être légèrement évaporées lors d'un séjour en surface car ce sont en partie des eaux du Lias qui se réinfiltrent pour apparaître ensuite au niveau du piémont. La corrélation entre les teneurs en oxygène- 18 et les teneurs en deutérium de quelques échantillons (fig. 2) montre que les points s'alignent selon la droite météorique mondiale (CRAIG, 1961). L'effet isotopique dû à l'évaporation est donc probablement négligeable devant celui induit par les variations des altitudes de recharge.

Tableau 2 Résultats des anảlysés isotopiques des sources de l'Atlas de Beni Mellal.

Table 2 Results of isotopic analysis of Beni Mellal Atlas springs.

\begin{tabular}{|c|c|c|c|c|c|c|c|c|}
\hline \multirow{3}{*}{$\begin{array}{c}\begin{array}{c}\text { Sources } \\
\text { échantilitonnées }\end{array} \\
\text { 1. Ain Asserdoune }\end{array}$} & \multirow{3}{*}{$\begin{array}{c}\text { No IAE } \\
210 / 37\end{array}$} & \multirow{3}{*}{$\begin{array}{c}\begin{array}{c}\text { Altitude } \\
\text { de la } \\
\text { source } \\
\text { (m) }\end{array} \\
647\end{array}$} & \multirow{3}{*}{$\begin{array}{c}\begin{array}{c}\text { Débit moyen } \\
\text { en } 1 / s\end{array} \\
1100\end{array}$} & \multirow{2}{*}{$\begin{array}{l}\text { Altitude } \\
\text { estimée } \\
\text { du BV } \\
\text { (en m) }\end{array}$} & \multicolumn{2}{|c|}{$\begin{array}{l}\text { Teneur en } \\
\text { Oxygène-18 } \\
\text { (delta \%o) }\end{array}$} & \multirow{2}{*}{$\begin{array}{l}\text { Deutérium } \\
\text { (delta \%o) } \\
\text { mars } 1993\end{array}$} & \multirow{2}{*}{$\begin{array}{l}\text { Formation } \\
\text { géologique } \\
\text { à l'exutoire }\end{array}$} \\
\hline & & & & & mars 93 & sep. 93 & & \\
\hline & & & & & $-7,5$ & $-7,5$ & $-48,6$ & Lias \\
\hline 2. Tamegnounte & $1505 / 37$ & 540 & 137 & $1350^{*}$ & $-6,7$ & $-7,4$ & $-42,5$ & Travertin Dir \\
\hline 3. Ourbiaå & $1885 / 37$ & 720 & 103 & $1360^{*}$ & $-6,5$ & $-6,5$ & - & Dir \\
\hline 4. Foum Oudi & $247 / 37$ & 540 & 35 & & $-5,8$ & $-6,1$ & - & " \\
\hline 5. Timoulilt & $249 / 37$ & 580 & 70 & $1260^{*}$ & $-6,3$ & $-6,9$ & $-37,1$ & $n$ \\
\hline 6. Ait Aâmira & & 1250 & 3 & & $-5,8$ & - & - & Bathonien \\
\hline 7. El ghazi & $1507 / 37$ & 670 & 5 & & $-5,9$ & - & - & Dir \\
\hline 8. S. Bouyakoub & $1565 / 37$ & 556 & 25 & $1300^{*}$ & - & $-7,2$ & - & • \\
\hline 9. Ksabi & $1770 / 37$ & 550 & 15 & $1250^{\star}$ & $-6,1$ & $-6,3$ & $-39,3$ & $"$ \\
\hline 10. Zitoune & $1773 / 37$ & 640 & 15 & & $-6,1$ & - & - & $"$ \\
\hline 11. Foum El ancer & $258 / 37$ & 650 & 125 & & $-5,0$ & $-6,7$ & - & " \\
\hline 12. Fechtalla & $1677 / 37$ & 695 & 124 & & $-5,4$ & - & $-34,4$ & $"$ \\
\hline 13. Fariata & $1393 / 37$ & 650 & 7 & & $-5,2$ & - & - & $"$ \\
\hline 14. Tazeroualte & $1959 / 37$ & 700 & 20 & $1050^{*}$ & $-5,7$ & $-6,6$ & - & ' \\
\hline 15. Tarnousdjoute & $1765 / 37$ & 694 & 57 & & - & $-7,7$ & - & Lias \\
\hline 16. Ain Oudaine & $1765 / 37$ & 640 & 143 & & $-5,5$ & $-6,6$ & - & Dir \\
\hline 17. Sidi B. Daoud & $1683 / 37$ & 680 & 69 & & $-5,1$ & - & - & $\cdot$ \\
\hline 18. Ouácha & $1499 / 37$ & 790 & 85 & & $-5,6$ & $-6,8$ & - & $"$ \\
\hline 19. Ousatrou & $1500 / 37$ & 820 & 175 & $1030^{*}$ & $-5,9$ & $-7,1$ & $-38,6$ & " \\
\hline 20. Gorm Nih & $1796 / 37$ & 565 & 11 & $1010^{*}$ & $-5,5$ & $-6,7$ & - & $"$ \\
\hline 21. Ait Daoud & $1800 / 37$ & 585 & 19 & $1020^{*}$ & $-5,7$ & $-6,6$ & - & * \\
\hline 22.Ouhriche & $1867 / 37$ & 680 & 20 & $860^{*}$ & $-5,1$ & $-6,1$ & & $\cdot$ \\
\hline 23. Taghbaioute & $1627 / 37$ & 1145 & 15 & $1360^{*}$ & $-6,4$ & $-7,4$ & $-41,8$ & Lias \\
\hline 24. Moha O'Khty & $1462 / 37$ & 1064 & 51 & & $-6,1$ & - & - & $"$ \\
\hline 25. Tamda amont & $361 / 37$ & 720 & 220 & & $-6,6$ & $-7,7$ & $-44,3$ & Lias \\
\hline 26. Tamda aval & & 700 & 100 & & $-6,7$ & - & - & " \\
\hline 27. Ouar n'łaá & & 600 & 120 & & - & $-7,6$ & - & * \\
\hline 28. Ain Sidi Said & & 1300 & 5 & & - & -7 & - & Crétacé Atlas \\
\hline
\end{tabular}

* Valeurs utilisées dans ta figure 3.

- Values used in figure 3. 


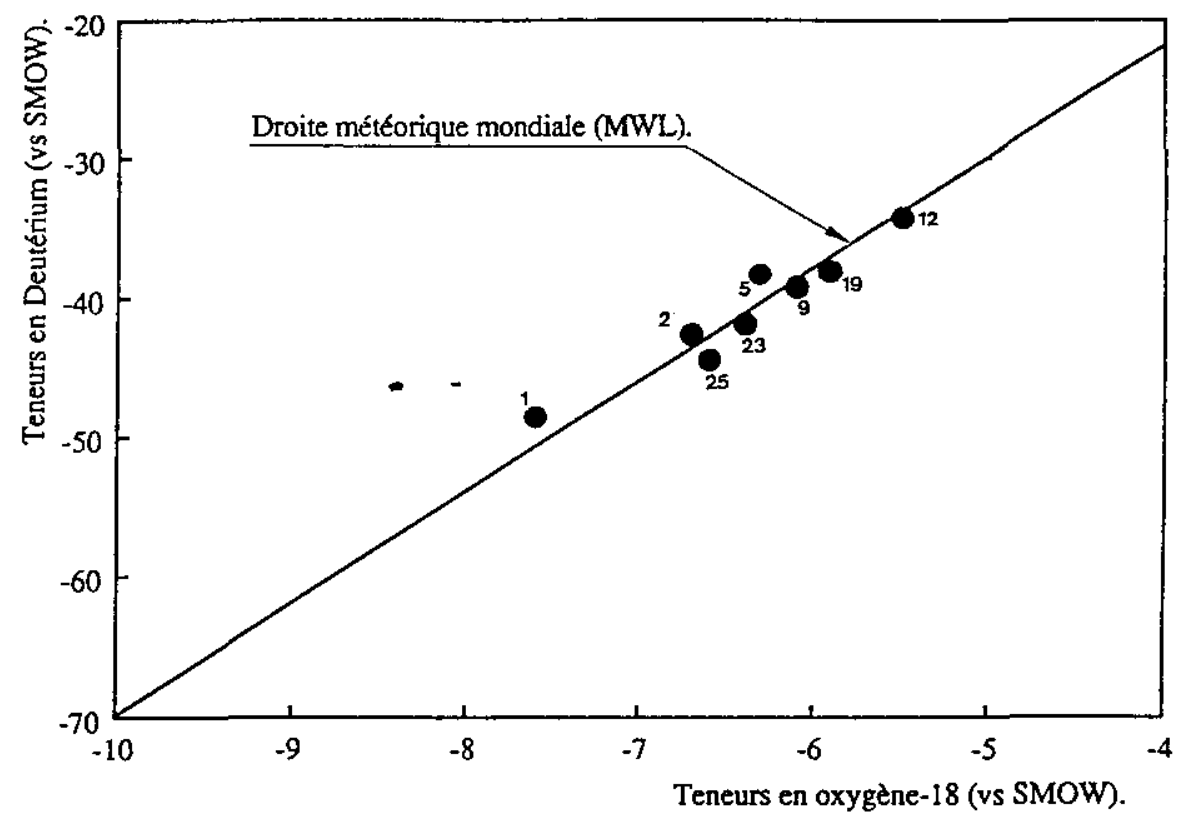

Figure 2 Relation entre teneurs en deutérium et teneurs en Oxygène-18 (mars 93).

Relationship between deuterium contents and oxygen-18 contents (March 93).

Le gradient de variation des teneurs en isotopes stables doit, en toute rigueur, être établi à partir des mesures sur les échantillons de précipitations collectées à différentes altitudes dans la région supposée de la recharge, et sur plusieurs années. Ne disposant pas de cette collection d'échantillons, nous avons tenté de vérifier la validité régionale du gradient en $0-18(0,3 \%$ 。 par $100 \mathrm{~m}$ ) proposé par MARCE (1975) et KABBAJ et al. (1978) et qui a été établi sur les précipitations dans les régions de l'Atlas.

$\mathrm{Vu}$ l'étendue du bassin d'alimentation des sources et la difficulté rencontrée pour délimiter le bassin versant de chacune d'elles, il est possible, d'après les profils topographiques et géologiques, d'estimer l'altitude moyenne du bassin d'alimentation. Cette estimation a été réalisée pour onze sources, réparties sur le chevauchement nord - atlasique sur une distance de $70 \mathrm{~km}$ et dont le bassin versant n'est à priori, ni trop tourmenté ni trop étendu. Les altitudes estimées ont ensuite été mises en relation avec les teneurs en oxygène-18 des eaux écoulées par les sources considérées, en mars et en septembre (fig. 3 ).

On remarque que les eaux de la campagne de mars s'alignent sur la droite de MARCE et pas celles de septembre. La variation de la teneur en 0-18 des eaux de sources prélevées en mars (période pluvieuse) correspond à un gradient altitudinal $0,26 \%$ par $100 \mathrm{~m}$ (fig. 3). En revanche, les points représentatifs des échantillons prélevés en septembre (période d'étiage) sont 
beaucoup plus dispersés. Les teneurs en oxygène- 18 sont bien corrélées aux altitudes moyennes de recharges pour les échantillons du printemps : $\delta \%$ $(0-18)=-3-0,0026 \times$ altitude, $r=0,96$ ( mars 93 : hautes eaux); elles ne le sont pas avec celles de l'automne.

II est difficile d'admettre que cette variation apparente du gradient puisse trouver son origine dans une variation saisonnière de la teneur en oxygène-18 des précipitations à une même altitude. Il semble donc que ce soit l'altitude de la recharge apparente qui varie avec la saison, ou plus vraisemblablement, avec l'état " hydraulique " des systèmes aquifères. Ainsi, en période de crue, les eaux écoulées aux sourcès semblent représenter un mélange des précipitations tombées sur l'ensemble du profil topographique de recharge. En période d'étiage, certaines sources pourraient drainer dans de plus grandes proportions des réserves d'origine plus lointaine, correspondant à des eaux précipitées à une altitude plus élevée que l'altitude moyenne du bassin versant.

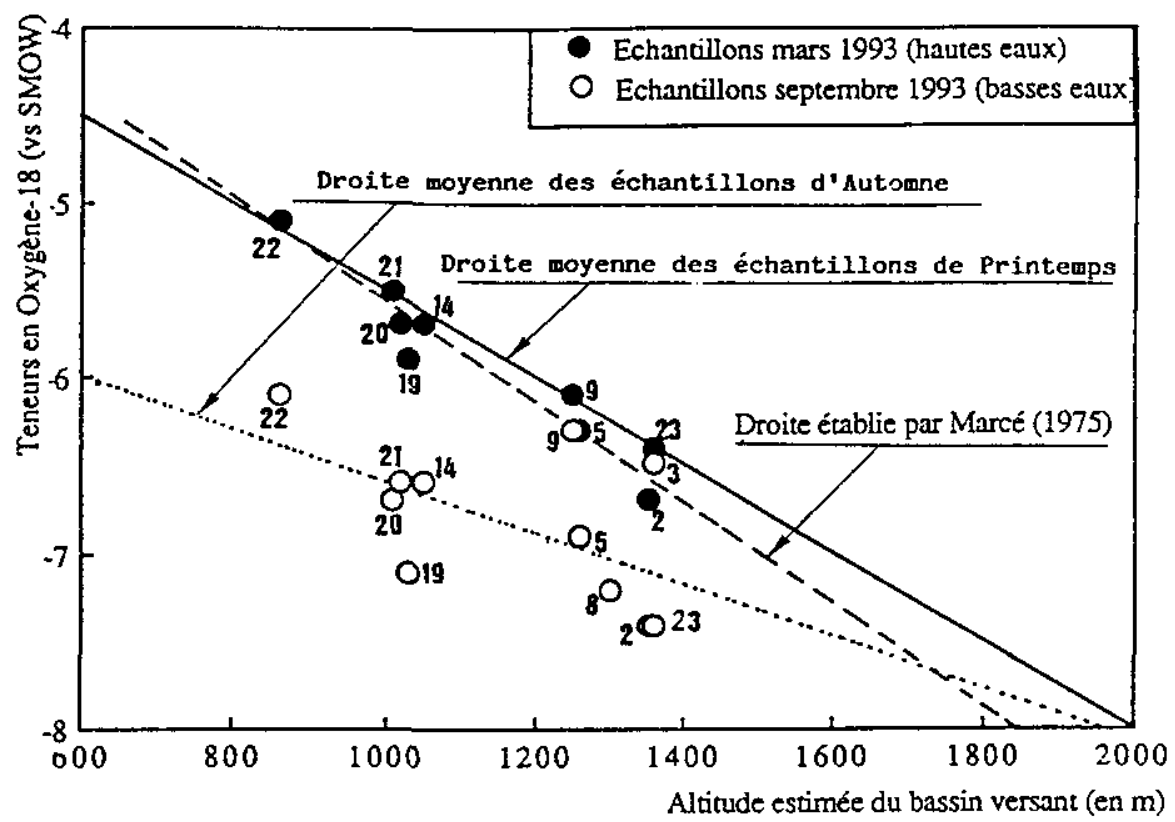

Figure 3 Relation entre teneurs en Oxygène-18 et altitudes moyennes du bassin versant.

Relationship between oxygen-18 contents and average altitude of the catchment basin.

Ces variations saisonnières dans l'alimentation sont particulièrement sensibles pour les sources présentant de faibles débits (fig. 4 et tabl. 2). Elles sont liées au mode de fonctionnement très inertiel du système (temps de régulation de 87 jours et coefficient de tarissement de f'ordre de $10^{-3}$, BOUCHAOU et al., 1994 à paraître). Les eaux des sources du Dir proviennent en général d'altitudes moyennes comprises entre 800 et $1200 \mathrm{~m}$ (moyens 
plateaux) en période de crue alors que des précipitations de plus hautes altitudes alimentent ces sources en période d'étiage (tabl. 3). Ces précipitations de hautes altitudes sont également à l'origine des eaux écoulées par les sources qui émergent dans le Lias, en période de crue comme en période d'étiage. Parmi les sources du Lias, l'exutoire principal de la région (Ain Asserdoune) ne subit pas de variations saisonnières; cet effet tampon (qui se trouve également dans les faibles variations de température enregistrées: 15,5 à $16^{\circ} \mathrm{C}$ ), témoigne de l'existence de réserves importantes. Les teneurs en oxygène-18 $(-7,6$ et $-7,5 \%)$, très proches en mars et en septembre, permettent de calculer une altitude de recharge moyenne supérieure à $1700 \mathrm{~m}$ correspondant aux massifs de Tacemit et Ighnyenne. Cette aftitude est plus

Tableau 3 Comparaison entre les altitudes moyennes de recharge estimées selon la topographie du bassin versant et calculées avec un gradient en $0-18$ de $0,26 \%$ par $100 \mathrm{~m}$.

Table 3 Comparison between average estimated altitude of intake area and calculated with an $0-18$ gradient like $2.6 \%$ per $1,000 \mathrm{~m}$.

\begin{tabular}{|c|c|c|c|c|c|c|}
\hline \multirow{2}{*}{$\begin{array}{c}\text { Sources } \\
\text { échantillonnées }\end{array}$} & \multicolumn{2}{|c|}{$\begin{array}{l}\text { Teneur en Oxygène-18 } \\
\text { delta \%o SMOW }\end{array}$} & \multirow{2}{*}{$\begin{array}{l}\text { Altitude topo. } \\
\text { estimée } \\
\text { du BV (en m) }\end{array}$} & \multicolumn{2}{|c|}{$\begin{array}{l}\text { Altitude de recharge } \\
\text { isotopique (en m) }\end{array}$} & \multirow{2}{*}{$\begin{array}{l}\text { Formation } \\
\text { géologique } \\
\text { à l'exutoire }\end{array}$} \\
\hline & mars 93 & sept. 93 & & mars 93 & sept. 93 & \\
\hline 1. Ain Asserdoune & $-7,6$ & $-7,5$ & & 1770 & 1730 & Lias \\
\hline 2. Tamegnounte & $-6,7$ & $-7,4$ & 1350 & 1430 & 1670 & Travertin Dir \\
\hline 3. Ourbiaâ & $-6,5$ & $-6,5$ & 1360 & 1330 & 1350 & Dir (piémont) \\
\hline 4. Foum Oudi & $-5,8$ & $-6,1$ & & 1080 & 1190 & " \\
\hline 5. Timoulilt & $-6,3$ & $-6,9$ & 1260 & 1280 & 1520 & $"$ \\
\hline 6. Ait Aâmira & $-5,8$ & - & & 1080 & & Bathonien \\
\hline 7. El ghazi & $-5,9$ & - & & 1120 & & Dir \\
\hline 8. S. Bounakoub & - & $-7,2$ & 1300 & & 1610 & " \\
\hline 9. Ksabi & $-6,1$ & $-6,3$ & 1250 & 1200 & 1270 & $\cdot$ \\
\hline 10. Zttoune & $-6,1$ & - & & 1200 & & " \\
\hline 11. Foum $E$ ancer & $-5,0$ & $-6,7$ & & 790 & 1420 & - \\
\hline 12. Fechtalla & $-5,4$ & - & & 950 & & * \\
\hline 13. Fariata & $-5,2$ & - & & 870 & & $\cdot$ \\
\hline 14. Tazeroualte & $-5,7$ & $-6,6$ & 1050 & 1060 & 1390 & $\cdot$ \\
\hline 15. Tamoudjoute & - & -7.7 & & & 1790 & Lias \\
\hline 16. Ain Oudaine & $-5,5$ & $-6,6$ & & 960 & 1400 & Dir \\
\hline 17. Sidi B. Daoud & $-5,1$ & - & & 820 & & " \\
\hline 18. Ouácha & $-5,6$ & $-6,8$ & & 1010 & 1480 & $\cdot$ \\
\hline 19. Ousafrou & $-5,9$ & $-7,1$ & & 1110 & 1570 & * \\
\hline 20. Gorm Nih & $-5,5$ & -6.7 & 1010 & 970 & 1440 & $\cdot$ \\
\hline 21. Ait Daoud & $-5,7$ & $-6,6$ & 1020 & 1040 & 1400 & * \\
\hline 22. Ouhriche & $-5,1$ & $-6,1$ & 860 & 810 & 1200 & $\cdot$ \\
\hline 23. Taghbaloute & $-6,4$ & $-7,4$ & 1360 & 1310 & 1680 & Lias \\
\hline 24. Moha O'Khty & $-6,1$ & - & & 1200 & & - \\
\hline 25. Tamda amont & $-6,6$ & $-7,7$ & & 1380 & 1800 & Lias \\
\hline 26. Tamda aval & $-6,7$ & - & & 1410 & & " \\
\hline 27. Ouar n'faâ & - & $-7,6$ & & & 1750 & ' \\
\hline 28. Ain Sidi Said & - & -7 & & & 1550 & Crétacé Atlas \\
\hline
\end{tabular}


élevée que celle fournie par les simples observations topographiques, ce qui montre le peu de fiabilité de ce type d'informations dès lors que le bassin versant atteint une certaine étendue.

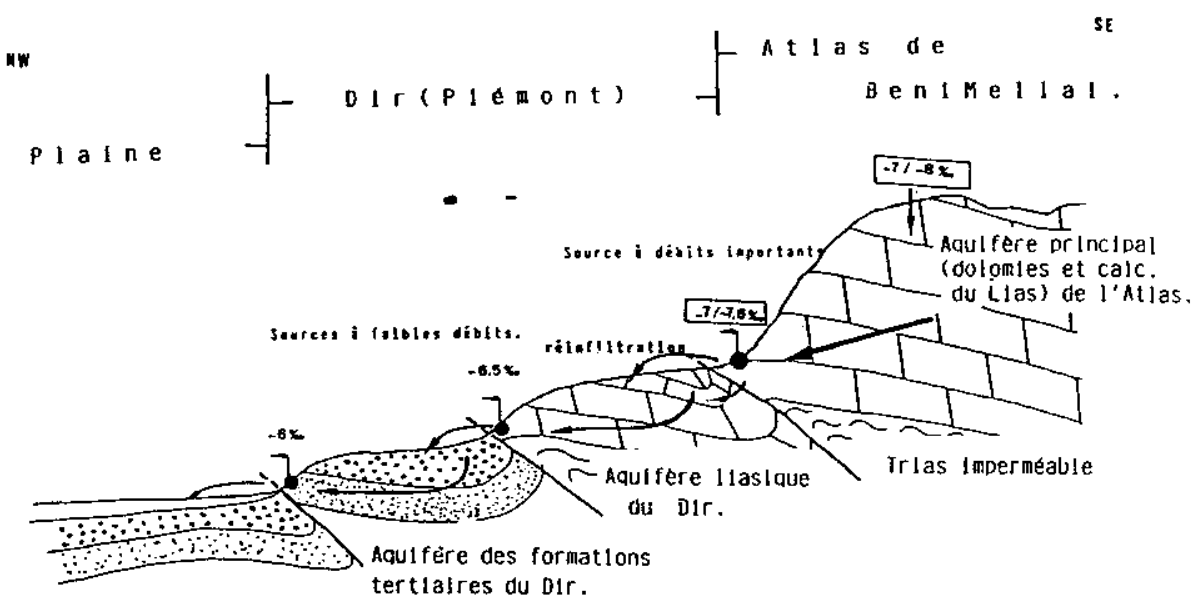

Figure 4 Schéma de circulation des eaux sur le piémont de l'Atlas de Beni Mellal. The water circulation diagram in piemont of Beni Mellal Atlas.

\section{4 - CONCLUSION}

L'analyse des résultats des traçages artificiels montre que les axes de drainage sont multiples, dont l'écoulement majeur converge vers la source d'Ain Asserdoune qui constitue une zone d'abaissement d'axe. Son bassin d'alimentation doit être étendu à l'ensemble du massif.

Les variations des teneurs en $0-18$ sont essentiellement interprétées en termes de différences d'altitudes de recharge. Le gradient altitudinal en 0-18, estimé à $0,26 \%$ par $100 \mathrm{~m}$, est très voisin de celui proposé par MARCE (1975) $(0,3 \%$ par $100 \mathrm{~m})$. Sa validité régionale est ainsi confirmée. Les précipitations sur les moyens plateaux $(800$ à $1200 \mathrm{~m}$ ) participent à l'alimentation des sources du Dir pendant les périodes de hautes eaux. Au contraire, ces sources sont majoritairement alimentées par les précipitations de haute altitude $(>1700 \mathrm{~m}$ ) en période d'étiage. Les variations saisonnières sont moins sensibles pour les sources du Lias et sont même totalement inexistantes pour l'exutoire principal du système, Ain Asserdoune, qui dispose probablement de réserves importantes, alimentées à une altitude moyenne supérieure à $1700 \mathrm{~m}$.

Par ailleurs, cette méthode confirme les résultats obtenus à partir des analyses hydrométriques qui ont montré un écoulement inertiel et complexe. Les résultats de ces campagnes isotopiques sur les sources de l'Atlas 
montrent l'utilité de cette technique isotopique pour la prospection d'une région karstique en zone semi-aride. Dans ce contexte climatique, l'existence d'étiages prolongés constitue une condition favorable à l'échantillonnage d'eaux représentatives de la réserve des systèmes, permettant la comparaison des altitudes d'infiltration de leur pluie efficace. Dans le contexte de Beni Mellal, la constance des teneurs en oxygène-18 a permis de confirmer l'inertie du système. Appliquée à d'autres sources, l'analyse des variations de teneurs en isotopes stables devrait permettre d'accéder directement au fonctionnement du système.

\section{REMERCIEMENTS}

Nous tenons à remercier l'Agence Internationale d'Energie Atomique (AIEA) pour l'aide financière attribuée à Mr Bouchaou, ce qui nous a permis d'effectuer les analyses isotopiques.

\section{RÉFÉRENCES BIBLIOGRAPHIQUES}

ARANYOSSY J.F., 1989. Quelques exemples pratiques d'application des isotopes de l'environnement aux études hydrogéologiques. Hydrogéologie, 3, 159-166.

BAKALOWICZ M., BLAVOUX B., MANGIN A., 1974. Apports du traçage isotopique naturel à la connaissance du fonctionnement d'un système karstique - Teneurs en oxygène-18 de trois systèmes des Pyrénées. France. J. Hydrol., 23, 141-158.

BLAVOUX B., MUDRY J., 1990. Les teneurs en oxygène-18 en fonction de l'altitude des impluviums des réservoirs karstiques du Jura et du Vaucluse. Mem. of the $22^{\text {nd }}$ Congress of $A l H, X X I I, 330-338$, Lausanne.

BLAVOUX B., BURGER A., CHAUVE P., MUDRY J., 1979. Utilisation des isotopes du milieu à la prospection hydrogéologique de la chaîne karstique du Jura. Rev. Geol. Dyn. Géog. Phys., 21, 4, 295-306, Paris.

BOUCHAOU L., 1988. Hydrogéologie du bassin des sources karstiques du complexe calcaire haut-atlasien du Dir de
Beni Mellal (Maroc). Doct. Univ. Franche Comte, 179 p - Besançon.

BOUCHAOU L., CHAUVE P., MANIA J., FERRANDIN! J., 1988. Structure et hydrologie de l'aquifère de l'Atlas de Beni Mellal. $4^{\theta}$ Coll. Hydro. en Pays Calc., Ann.. Scientif. Univ. Besançon, Géol. H.S, 6, 211-220.

BOUChaOU L., ChaUVE P., MANIA J., MANGIN A., ABOUFIRASSI M., 1994. Contribution à l'étude du fonctionnement hydrodynamique d'un système aquifère karstique en zone-aride : exemple de l'Atlas de Beni Mellal (Maroc).Gèdynamica Acta (à paraître).

CHAUVE P., MUDRY J., ROSENTHAL P., TISSOT, TRESSE Ph., 1986. Rôle des abaissements d'axe dans les circulations karstiques du Jura. Bull. Soc. Géol. France t. II, n'2, pp. 329-336.

CRAIG H., 1961. Isotopic variations in meteoric waters. Science, vol. 133, p. 1702-1703. 
FONTES J.Ch., 1976. Isotopes du milieu et cycles des eaux naturelles: quelques aspects. Thèse Doct. Etat, ès Sc. Nat. Univ. P. et M. Curie, Paris VI.

IAEA, 1981. Stable isotope Hydrology Deuterium and Oxygen-18 in water cycle. Technical reports series 210 , Vienna (Austria).

KABBAJ A., ZAYOUHI 1., CARLIER Ph., MARCE A., 1978. Contribution des isotopes du milieu à l'étude des grands aquifères du Maroc. Isot. Hydrol.. IAEA, II, SM., 228/25, Vienne (Autriche).

MANGIN A., 1975. Contribution à l'étude hydrodynamique des aquifères karstiques. Thèse Doct. Sc. Nat. Dijon, France.
MARCE A., 1975. Contribution des méthodes isotopiques à l'étude des modalités d'alimentation et de renouvellement des réserves souterraines du Maroc. SGN. 447, LAB. B.R.G.M., Orléans.

MOLINARI J., 1977. Quelques remarques pratiques et une présentation commode facilitant l'exploitation des variations naturelles en deutérium et en oxygène-18 dans les eaux. J. of Hydrol , 32, pp. 383-392.

MONBARON M., 1982. Précisions sur la chronologie de la tectogénèse atlasique. C. R. Acad. Sc. Paris, 294, II : 883-885.

MONBARON M., 1985. Carte géologique du Maroc au 1/100 000. Feuille de Beni Mellal. Notes et Mém. Serv. Géol. Maroc, 341. Rabat. 